\title{
Fatores de risco cardiovascular em estudantes da Faculdade de Ciências Médicas de Campina Grande
}

\section{Cardiovascular risk factors in students from the health Sciences Center - Campina Grande - Brazil}

Guilherme Veras Mascena ${ }^{1}$, Manuela Stefania Barros Cavalcante ${ }^{2}$, Gustavo Brasil Marcelino², Samara de Aquino Holanda $^{2}$, Carlos Teixeira Brandt ${ }^{3}$

\begin{abstract}
RESUMO
Fundamento: Pesquisa relacionada a fatores de risco cardiovascular entre universitários é essencial, considerando seus papéis na sociedade.

Objetivos: Avaliar os fatores de risco cardiovascular entre estudantes da área de saúde de uma Universidade de Campina Grande.

Materiais e métodos: Foi realizado estudo envolvendo 234 universitários com média das idades de 23,2 $\pm 3,9$ anos. Os estudantes foram entrevistados, foram obtidos os dados antropométricos e aferida a pressão arterial. As variáveis contínuas foram expressas por suas médias e desvios padrão, enquanto as categóricas por suas freqüências. Foi usado o teste t de Student visando identificar diferenças entre médias. O teste do qui-quadrado foi utilizado para avaliar diferenças entre freqüências. O nível de significância foi $p<0,05$. O estudo foi aprovado no Comitê de Ética.

Resultados: A maioria (161 - 68,8\%) dos universitários foi do sexo feminino. O curso de medicina respondeu pelo maior número $(150-64,1 \%)$ de graduandos pesquisados. Os cursos de fisioterapia e enfermagem responderam respectivamente por $17,9 \%$ dos alunos incluídos no estudo. A maioria (53,4\%) dos pais dos alunos tinha curso superior completo. Sedentarismo foi relatado por $123(52,6 \%)$ alunos. A prevalência de sobrepeso e obesidade foi de 43,6\%. Hipertensão arterial sistêmica (HAS) foi verificada em 37 (15,8\%) estudantes; todavia, em apenas cinco (14,7\%) a doença estava sob controle.

Conclusão: As prevalências de: sobrepeso/obesidade e sedentarismo foram altos $( \pm 40 \%)$ estando associados à HAS.
\end{abstract}

Palavras-chave: Fatores de Risco / Sistema Cardiovascular. Estudantes/Universidades. Atividade Física. Universidades.

\section{Introdução}

As doenças cardiovasculares (DCV) constituem a principal causa de mortalidade no Brasil $^{1}$ e no mundo ${ }^{2-5}$ e seus fatores de risco podem ser divididos em duas categorias: fatores de risco modificáveis como hipertensão arterial sistêmica (HAS) ${ }^{6}$, diabetes mellitus $(\mathrm{DM})^{7}$, dislipidemia (DLP) ${ }^{8}$, tabagismo ${ }^{5}$, obesida$\mathrm{de}^{9} \mathrm{e}$ sedentarismo e fatores de risco não modificáveis como idade, sexo, raça e hereditariedade.

O processo aterosclerótico, responsável pelo desenvolvimento das DCV, se inicia já nas primeiras
1. Especialista em Cardiologia pela Sociedade Brasileira de Cardiologia

2. Acadêmicos da Faculdade de Ciências Médicas de Campina Grande

3. PhD, Assessor Científico da Faculdade de Ciências Médicas de Campina Grande
Correspondência:

Avenida Floriano Peixoto 1650, apto 1203, Alto Branco, Campina Grande-PB, CEP 58400-180 guilherme.mascena@globo.com

Artigo recebido em 27/02/2012 Aprovado para publicação em 20/06/2012 
décadas de vida e se acompanha de um longo período de latência que pode ser abreviado pela exposição aos maus hábitos de vida ${ }^{10}$. No Brasil, tem-se observado elevada prevalência de fatores risco cardiovascular (FRCV) entre os adultos jovens especialmente de sedentarismo e obesidade. ${ }^{1}$

O impacto sócio-econômico das DCV tem estimulado a implantação de políticas públicas de conscientização da população acerca dos principais fatores de risco cardiovasculares. Para os jovens, em particular, a mudança do estilo de vida pode alterar a história natural da doença aterosclerótica. ${ }^{11}$

Entre os universitários, particularmente da área de saúde, se observa elevada prevalência de FRCV que é preocupante já que formam importante grupo de quem se espera modelo para o estabelecimento de hábitos de vida saudáveis na população geral. ${ }^{12-17}$ Este estudo teve como objetivo estabelecer a prevalência de obesidade, sedentarismo, hipertensão arterial e tabagismo numa população de estudantes do ensino superior na área de Saúde.

\section{Procedimentos metodológicos}

O estudo observacional, transversal, randomizado envolveu alunos da Faculdade de Ciências Médicas de Campina Grande (FCM-CG), instituição privada, dos cursos de Medicina, Enfermagem e Fisioterapia. Para o cálculo do tamanho da amostra foram adotados os seguintes critérios: a) população finita de 1105 estudantes das três Faculdades; b) prevalência estimada de hipertensão arterial em $18 \% \pm 5 \%$; c) intervalo de confiança de $97 \%$; d) erro amostral de 5\%; e) acréscimo de $20 \%$ para possíveis perdas ou recusas. Foram incluídos 234 voluntários de ambos os sexos com idades entre 19 e 37 anos com média de 23,2 $\pm 3,9$ anos. Foram excluídas as alunas grávidas.

Os alunos foram convidados pessoalmente e através de contato telefônico, sendo entrevistados e examinados na Clínica Escola da FCM-CG. Foram preenchidos questionários estruturados e divididos em dados gerais de identificação, grau de instrução dos pais, antecededentes familiares de FRCV, dados sobre a atividade física, medidas antropométricas e de pressão arterial. Foram considerados os antecedentes familiares que envolveram parentes de primeiro grau com eventos precoces: antes de 65 anos de idade em parentes de primeiro grau do sexo feminino e antes dos 55 anos de idade, em parentes do masculino.

Para avaliação do nível de atividade física os estudantes responderam ao questionário International
Physical Activity Questionnaire versão 8 (IPAQ-8), forma curta, validada no Brasil. ${ }^{18}$ A pontuação foi obtida pela soma da quantidade de dias e minutos ou horas das atividades físicas realizadas na semana anterior ao preenchimento do questionário. Segundo o IPAQ-8 e considerando os critérios freqüência, intensidade e duração, os níveis de atividade física se dividem em 5 categorias: muito ativo, ativo, insuficientemente ativo A, insuficientemente ativo B e sedentário. No presente estudo, para efeito de procedimentos analíticos, foi feita estratificação com apenas três categorias: a) ativos, que correspondem à soma das categorias muito ativo e ativo do IPAQ-8, b) insuficientemente ativos, que agrega os insuficientemente ativos A e B, e c) sedentários.

As determinações de peso (P), altura (A) e índice de massa corpórea (IMC) foram realizadas com os alunos descalços, usando roupas leves numa balança calibrada de marca Welmy, seguindo as recomendações do Manual de Técnicas e Procedimentos do Ministério da Saúde. ${ }^{19} \mathrm{O}$ estado nutricional global foi determinado segundo o IMC com base nos limites propostos pela Organização Mundial de Saúde $(\mathrm{OMS})^{20}$ sendo definido baixo peso IMC $<18,5 \mathrm{Kg} /$ $\mathrm{m}^{2}$, normal como IMC $\geq 18,5 \mathrm{e}<25 \mathrm{Kg} / \mathrm{m}^{2}$, sobrepeso como IMC $\geq 25$ e $<30 \mathrm{Kg} / \mathrm{m}^{2}$ e obesidade como $\mathrm{IMC} \geq 30 \mathrm{Kg} / \mathrm{m}^{2}$.

As determinações da circunferência abdominal e do quadril foram realizadas com fita métrica inelástica. A medida da circunferência abdominal (CA) foi determinada no ponto médio entre a crista ilíaca e a última costela. Foram utilizados os limites de corte propostos pela OMS que considera adequada uma CA $<80 \mathrm{~cm}$ para mulheres e $<94 \mathrm{~cm}$ para homens e estabelece como limites de risco 1 (risco aumentado) uma CA entre 94 e $102 \mathrm{~cm}$ para homens e entre 80 e $88 \mathrm{~cm}$ para mulheres e risco 2 (risco muito aumentado) acima de $102 \mathrm{~cm}$ para homens e $88 \mathrm{~cm}$ para mulheres. ${ }^{20}$ A medida da circunferência do quadril foi obtida na linha transtrocantérica. Foi determinada a relação cintura/quadril e considerada aumentada quando acima de 0,9 para homens e 0,8 para mulheres.

Para medida da pressão arterial foi utilizado o esfigmomanômetro OMRON HEM 705 CP, eletrônico, oscilométrico, validado e recomendado pelas instituições internacionais competentes. ${ }^{21,22}$ As medidas foram efetuadas com o entrevistado sentado, pés apoiados ao chão, braço esquerdo relaxado, apoiado sobre mesa e à altura do coração, palma voltada para cima, bexiga vazia, sem ter praticado exercícios moderados ou pesados, fumado ou ingerido bebida alco- 
ólica nos 30 minutos antecedentes às medidas, sendo usada braçadeira compatível com a circunferência do braço. Duas medidas foram obtidas separadas por intervalo de cinco minutos. Foi considerada a menor das aferições obtidas. Seguindo as recomendações da Sociedade Brasileira de Cardiologia foram considerados hipertensos os indivíduos que apresentassem cifras sistólicas iguais ou superiores a $140 \mathrm{mmHg}$ e/ou cifras diastólicas iguais ou superiores a $90 \mathrm{mmHg}$. Aqueles cujas cifras sistólicas se encontrassem entre 135 e 139 $\mathrm{mmHg}$ e/ou as diastólicas se situassem entre 85 e 89 $\mathrm{mmHg}$ foram categorizados como pré hipertensos. ${ }^{23}$

Os dados foram armazenados em planilhas de Excel e a análise foi feita usando o SPSS versão 18.0.

As variáveis quantitativas foram expressas por suas médias e desvios-padrão. As variáveis qualitativas foram expressas por suas freqüências absolutas e relativas. Foi usado o teste t de Student para amostras independentes visando identificar possíveis diferenças entre médias. O teste do qui-quadrado foi utilizado para avaliar possíveis diferenças entre freqüências.O nível de rejeição estabelecido para a hipótese de nulidade foi menor ou igual a $0,05(5 \%)$.

O estudo foi aprovado pelo Comitê de Ética e Pesquisa do Centro de Ensino Superior e Desenvolvimento (CEP/CESED) e seguiu as diretrizes da resolução no 196/96 do Conselho Nacional de Saúde. Todos os indivíduos que participaram do estudo assinaram o Termo de Consentimento Livre e Esclarecido (TCLE). ${ }^{24}$

\section{Resultados}

Foram examinados 73 homens $(31,2 \%)$ e 161 mulheres $(68,8 \%)$. A faixa etária variou de 18 a 37 anos com média de idade de 23,2 $\pm 3,9$ anos. (Tabela 1). Os alunos de Medicina constituíram a maioria da amostra com 150 entrevistados $(64,1 \%)$ e os alunos de Enfermagem e Fisioterapia compuseram os demais com 42 entrevistados $(17,9 \%)$ de cada curso o que refletiu as proporções de indivíduos que compõem as três Faculdades. A maioria $(52,4 \%)$ dos pais dos alunos tinha curso superior completo.

A média dos IMC foi $24,9 \pm 4,1 \mathrm{Kg} / \mathrm{m}^{2}$, variando de 16,4 a $42,5 \mathrm{Kg} / \mathrm{m}^{2}$. As médias das pressões arterial sistólica (PAS) e diastólica (PAD) foram: 119,3 $\pm 15,6 \mathrm{mmHg}$ e $73,5 \pm 9,5 \mathrm{mmHg}$, respectivamente. As médias das PAS, PAD e PAM foram significantemente maiores entre os homens quando comparadas com as das mulheres (Homens - 132,0 $\pm 13,4 \mathrm{mmHg}, 76,0$ $\pm 9,2 \mathrm{mmHg}$ e $94,7 \pm 9,3 \mathrm{mmHg}$ versus Mulheres $113,6 \pm 13,0 \mathrm{mmHg}, 72,4 \pm 9,5 \mathrm{mmHg}$ e $86,1 \pm 10,1$ mmHg - p<0,0001). (Tabela 2)

A prevalência de HAS foi de $15,8 \%$ sendo maior no sexo masculino $(34,2 \%$ versus $6,2 \%, \mathrm{p}<0,0001)$. Embora o relato de HAS na família tenha sido referido em apenas 11 entrevistados, ele esteve fortemente associado à presença de HAS com um risco relativo de 7,5 (variando entre 5,0 a 11,2 para um intervalo de confiança de $95 \%$ ). O estágio de pré-hipertensão foi detectado em 26 entrevistados $(11,1 \%)$ com predomí-

\section{Tabela 1}

Distribuição das médias e desvios-padrão das características da população, segundo sexo

\begin{tabular}{|c|c|c|c|c|c|c|}
\hline \multirow[b]{2}{*}{ Variável } & \multicolumn{2}{|r|}{ Geral } & \multicolumn{2}{|c|}{ Masculino } & \multicolumn{2}{|c|}{ Feminino } \\
\hline & Média & Desvio padrão & Média & Desvio padrão & Média & Desvio padrão \\
\hline Idade (anos) & 23,3 & 3,9 & $24,28^{*}$ & 3,6 & 23,0 & 3,9 \\
\hline Altura $(\mathrm{cm})$ & 165,9 & 8,9 & $175,1^{\dagger}$ & 6,5 & 161,7 & 6,4 \\
\hline Peso (Kg) & 69,0 & 15,8 & $82,2^{\dagger}$ & 15,4 & 63,0 & 11,0 \\
\hline $\operatorname{IMC}\left(\mathrm{Kg} / \mathrm{m}^{2}\right)$ & 24,9 & 4,1 & $26,8^{\dagger}$ & 4,3 & 24,0 & 3,7 \\
\hline $\mathrm{CA}(\mathrm{cm})$ & 84,3 & 12,3 & $91,6^{\dagger}$ & 11,6 & 80,8 & 11,0 \\
\hline PAS (mmHg) & 119,3 & 15,6 & $132,0^{\dagger}$ & 13,4 & 113,6 & 13,0 \\
\hline $\mathrm{PAD}(\mathrm{mmHg})$ & 73,5 & 9,5 & $76,0^{*}$ & 9,2 & 72,4 & 9,5 \\
\hline $\mathrm{PAM}(\mathrm{mmHg})$ & 88,8 & 10,6 & $94,7^{\dagger}$ & 9,3 & 86,1 & 10,1 \\
\hline
\end{tabular}

${ }^{*} p=0,023$ em relação ao sexo feminino (teste $t$ ), ${ }^{\dagger} p<0,0001$ em relação ao sexo feminino (teste $t$ ), ${ }^{\ddagger} p=0,007$ em relação ao sexo feminino (teste t)

IMC - índice de massa corpórea, CA - circunferência abdominal, PAS - pressão arterial sistólica, PAD - pressão arterial diastólica,

PAM - pressão arterial média, ANTEC HAS - antecedentes familiares de hipertensão arterial 
Tabela 2

Distribuição das frequências absoluta e relativa das variáveis hipertensão arterial, sedentarismo, obesidade e tabagismo segundo o sexo

\begin{tabular}{|c|c|c|c|c|c|c|c|}
\hline \multirow[b]{2}{*}{ Variável } & & \multicolumn{2}{|r|}{ Geral } & \multicolumn{2}{|r|}{ Masculino } & \multicolumn{2}{|r|}{ Feminino } \\
\hline & & $\mathrm{N}^{\circ}$ & Frequência (\%) & $\mathrm{N}^{\mathrm{o}}$ & Frequência (\%) & $\mathrm{N}^{\mathrm{o}}$ & Frequência (\%) \\
\hline \multirow[t]{2}{*}{ Pré HAS } & Presente & 26 & 11,1 & 16 & $21,9^{*}$ & 10 & 6,2 \\
\hline & Ausente & 208 & 88,9 & 57 & 78,1 & 151 & 93,8 \\
\hline \multirow[t]{2}{*}{ HAS } & Presente & 37 & 15,8 & 25 & $34,2^{\dagger}$ & 12 & 6,2 \\
\hline & Ausente & 197 & 84,2 & 48 & 65,8 & 149 & 93,8 \\
\hline \multirow[t]{3}{*}{ Atividade Física } & Ativo & 77 & 32,9 & 31 & $42,5^{\prime \prime}$ & 46 & 28,6 \\
\hline & Insuf. Ativo & 34 & 14,5 & 15 & 20,5 & 19 & 11,8 \\
\hline & Sedentário & 123 & 52,6 & 27 & 37,0 & 96 & 59,6 \\
\hline \multirow[t]{4}{*}{ Obesidade } & $\mathrm{IMC}<18,5$ & 6 & $2,6^{\ddagger}$ & 1 & 1,4 & 5 & 3,1 \\
\hline & $\geq 18,5$ ou $<25$ & 126 & 53,8 & 22 & 30,1 & 104 & 64,6 \\
\hline & $\geq 25$ ou $<30$ & 80 & 34,2 & 39 & 53,4 & 41 & 25,5 \\
\hline & $\geq 30$ & 22 & 9,4 & 11 & 15,1 & 11 & 6,8 \\
\hline \multirow[t]{3}{*}{ CA } & Normal & 96 & 41,0 & 24 & 32,9 & 72 & 44,7 \\
\hline & Risco I & 101 & 43,2 & 37 & 50,7 & 64 & 39,8 \\
\hline & Risco II & 37 & 15,8 & 12 & 16,4 & 25 & 15,5 \\
\hline \multirow[t]{2}{*}{ RCQ } & Normal & 135 & 57,7 & 46 & 63,0 & 89 & 55,3 \\
\hline & Aumentada & 99 & 42,3 & 27 & 37,0 & 72 & 44,7 \\
\hline \multirow[t]{2}{*}{ Tabagismo } & Presente & 15 & 6,4 & 11 & $15,1^{\S}$ & 4 & 2,5 \\
\hline & Ausente & 219 & 93,6 & 62 & 84,9 & 157 & 97,5 \\
\hline
\end{tabular}

* $p=0,0011,{ }^{\dagger} p<0,0001, " / p=0,0007$, todos em relação ao sexo feminino pelo teste exato de Fischer;

\& $p<0,0001,{ }^{\ddagger} p=0,0053$, para o teste quiquadrado em relação ao sexo feminino

Pré HAS - pré hipertenso, HAS - hipertensão arterial sistêmica, Insuf. Ativo - insuficientemente ativo, CA - circunferência abdominal, $\mathrm{RCQ}$ - relação cintura/quadril

nio entre os homens $(21,9 \%$ versus $6,2 \%, p=0,0011)$. Em cinco $(14,7 \%)$ dos universitários hipertensos a doença estava controlada com PAS $<140 \mathrm{mmHg}$ e $\mathrm{PAD}<90 \mathrm{mmHg}$.

Sedentarismo esteve presente em $123(52,6 \%)$ dos entrevistados e foi igualmente prevalente em ambos os sexos. Quinze $(6,4 \%)$ desses referiram o hábito de fumar regularmente. Obesidade esteve presente em $22(9,4 \%)$ indivíduos e sobrepeso em $80(34,2 \%)$. (Tabela 2)

Foram observadas associações significantes entre HAS e obesidade $(\mathrm{p}<0,0001), \mathrm{CA}(\mathrm{p}<0,0001)$ e ao hábito de fumar $(\mathrm{p}=0,0178)$. (Tabela 3 )

\section{Discussão}

Em estudos epidemiológicos tem sido observada tendência de aumento da incidência das DCV em todo o mundo, particularmente nos países em desenvolvimento. ${ }^{25,26}$

Além das outras variáveis antropométricas, a distribuição da gordura é também um importante fator de risco para as doenças ligadas à obesidade. $\mathrm{O}$ excesso de gordura abdominal (também chamada de obesidade central ou visceral) está particularmente associado ao risco aumentado de doenças cardiometabólicas ${ }^{27}$, embora sua presença não melhore de for- 


\section{Tabela 3}

Distribuição das frequências absoluta e relativa das variáveis sedentarismo, obesidade, circunferência abdominal e tabagismo, segundo a presença de hipertensão arterial

\begin{tabular}{|c|c|c|c|c|c|}
\hline \multirow[b]{2}{*}{ Variável } & & \multicolumn{2}{|c|}{ Normotensão } & \multicolumn{2}{|c|}{ HAS } \\
\hline & & Número & Frequência (\%) & Número & Frequência $(\%)$ \\
\hline \multirow[t]{3}{*}{ Atividade Física } & Ativo & 68 & 34,5 & 9 & 24,3 \\
\hline & Insuf. Ativo & 23 & 11,7 & 11 & 29,7 \\
\hline & Sedentário & 106 & 53,8 & 17 & 46,0 \\
\hline \multirow[t]{4}{*}{ Obesidade } & $\mathrm{IMC}<18,5$ & $6^{*}$ & 3,0 & 0 & 0 \\
\hline & $>=18,5$ ou $<25$ & 119 & 60,4 & 7 & 18,9 \\
\hline & $>=25$ ou $<25$ & 62 & 31,5 & 18 & 48,7 \\
\hline & $>=30$ & 10 & 5,1 & 12 & 32,4 \\
\hline \multirow[t]{3}{*}{ CA } & Normal & $114^{\dagger}$ & 57,9 & 10 & 27,0 \\
\hline & Risco I & 58 & 29,4 & 11 & 29,8 \\
\hline & Risco II & 25 & 12,7 & 16 & 43,2 \\
\hline \multirow[t]{2}{*}{ RCQ } & Normal & 116 & 58,9 & 19 & 51,4 \\
\hline & Aumentado & 81 & 41,1 & 18 & 48,6 \\
\hline \multirow[t]{2}{*}{ Tabagismo } & Presente & $9:$ & 4,6 & 6 & 16,2 \\
\hline & Ausente & 188 & 95,4 & 31 & 83,8 \\
\hline
\end{tabular}

${ }^{*} p<0,0001$ para a comparação das frequências entre normotensos e hipertensos pelo teste quiquadrado; ${ }^{\dagger} p<0,0001 e^{\ddagger} p=0,0178$ para o teste exato de Fischer

$\mathrm{CA}$ - circunferência abdominal, Insuf. Ativo - insuficientemente ativo, RCQ - relação cintura/quadril

ma significativa a predição de risco quando são conhecidas as variáveis de pressão arterial, glicemia e perfil lipídico do indivíduo. ${ }^{28}$

Em que pese a baixa média de idade da população aqui estudada (23,2 $\pm 3,9$ anos) foram elevadas as prevalências de sobrepeso $(34,2 \%)$, obesidade $(9,4 \%)$, HAS $(15,8 \%)$ e, sobretudo, sedentarismo $(52,6 \%)$.

A prevalência elevada de sedentarismo no estudo atual se encontra concordante com outros estudos que analisaram população semelhante ${ }^{12-17}$, embora a variação seja ampla de $13,8 \%$ a $78,9 \%$ o que provavelmente reflete a utilização de critérios diversos para classificar o indivíduo como fisicamente ativo. No presente estudo, a utilização de três estratos para análise da atividade física buscou uma melhor caracterização do estrato intermediário entre aqueles completamente sedentários e os regularmente ativos. Há uma clara relação dose-resposta entre atividade física e o risco de DCV. Quantidades crescentes de atividade física estão associadas a reduções adicionais de risco tanto em homens como em mulheres, embora a forma da curva dose-resposta não esteja bem definida. ${ }^{29}$ Em estudos observacionais prospectivos, além da redução de risco cardiovascular, a atividade física regular se mostra inversamente relacionada com o aparecimento de osteoporose, câncer de cólon, câncer de mama, ansiedade e depressão. ${ }^{30}$

A relação entre HAS e risco cardiovascular está bem estabelecida na literatura. ${ }^{2,6}$ A prevalência aqui encontrada $(15,8 \%)$ se situou na média de estudos semelhantes onde se podem constatar prevalências que variam de $9,7 \%$ a $23,5 \%{ }^{12-16}$ sendo também significativa a prevalência encontrada de indivíduos em préhipertensão $(11,1 \%)$. O predomínio do sexo masculino entre os indivíduos hipertensos e pré-hipertensos reproduz os achados de outras séries no Brasil. ${ }^{12,13}$ 
As frequências de sobrepeso e obesidade deste estudo estiveram no limite superior de outras séries com populações semelhantes. ${ }^{12-17}$ Isto talvez seja reflexo da elevada prevalência de sedentarismo aqui encontrada já que esta associação é bastante conhecida. ${ }^{31} \mathrm{Um}$ dos fatores que pode contribuir para o sedentarismo nos estudantes universitários da área de saúde é o exíguo tempo livre das atividades ligadas à Faculdade. Esta situação é relevante ao pensar que, por serem estudantes da área de saúde, estes têm o conhecimento da importância da prática regular de atividade física na qualidade de vida e na promoção da saúde, atuando inclusive como fator protetor contra o aparecimento de doenças cardiovasculares. Adicionalmente, o exemplo pessoal pode, no futuro, servir de modelo para os indivíduos e pacientes que estarão sob seus cuidados.

Algumas limitações podem ser apontadas nesse estudo. A principal delas foi não dimensionar o percentual de usuários de álcool já que essa é uma variável de confundimento associada à presença de HAS e à obesidade..$^{32}$ Não obstante, vários estudos demonstraram que a associação entre HAS e obesidade se faz de forma independente e parece ter no seu cerne a ativação do sistema nervoso simpático e a retenção primária de sódio. ${ }^{33}$ Outra limitação foi não ter investigado as razões ligadas ao sedentarismo e a não observância dos hábitos alimentares dos entrevistados o que impede que se determine o impacto destes hábitos no peso dos estudantes.
Uma vez que obesidade, sedentarismo, tabagismo e HAS são fatores de risco modificáveis é desejável a promoção de programas que motivem a adoção de hábitos saudáveis dentro do próprio ambiente universitário já que esses estudantes são poderosos agentes multiplicadores para toda a população. Além do mais, reforça a necessidade da criação de serviço médico para avaliação do estado de saúde dos universitários entrantes no primeiro período, assim como para acompanhamento médico ao longo dos seus cursos. A instalação de um Serviço de Saúde para o Estudante, onde poderão atuar vários profissionais da área de saúde como: clínicos, cardiologistas, endocrinologistas, nutricionistas, fisioterapeutas e educadores físicos, acrescentará mais humanismo e prática preventiva para futuras doenças e agravos, melhorando a qualidade da Instituição.

\section{Conclusão}

As prevalências de: sobrepeso/obesidade e sedentarismo foram altos $( \pm 40 \%)$ estando associados à HAS.

Potencial Conflito de Interesses: Declaro não haver conflitos de interesses pertinentes

Fontes de Financiamento: O presente estudo não teve fontes de financiamento externas

Vinculação Acadêmica: Não há vinculação deste estudo a programas de pós-graduação

\begin{abstract}
Background: Research related to cardiovascular risk factors among college students is essential, considering their roles in society.

Purpose: To assess the cardiovascular risk factors among students of the Health Sciences of a University of Campina Grande.

Materials and methods: We conducted a study involving 234 university students with mean age of 23.2 \pm 3.9 years. Students were interviewed, obtained the anthropometric data and measured blood pressure. Continuous variables were expressed as means and standard deviations, while categorical variables by their frequencies. We used the Student $t$ test to identify differences between means. The chi-square test was used to assess differences between frequencies. The level of significance was $p<0.05$. The study was approved by the Ethics Committee.

Results: The majority (161 to $68.8 \%$ ) of students were female. The medical school accounted for the largest number (150-64.1\%) of undergraduates surveyed. The courses of physiotherapy and nursing respectively accounted for $17.9 \%$ of students enrolled in the study. Most $(53.4 \%)$ of parents had completed college. Sedentary lifestyle was reported by $123(52.6 \%)$ students. The prevalence of overweight and obesity was $43.6 \%$. Hypertension (HBP) was observed in 37 (15.8\%) students, however, in only five $(14.7 \%)$ the disease was under control.
\end{abstract}

Conclusion: The prevalence of: overweight / obesity and physical inactivity were high $( \pm 40 \%)$ and are associated with hypertension.

Keywords: Risk Factors / Cardiovascular System. Students /Universities. Physical Activity. Universities. 


\section{Referências}

1. Mansur AP, Lopes AIA, Favarato D, et al. Transição epidemiológica da mortalidade por doenças circulatórias no Brasil. Arq Bras Cardiol. 2005;93:506-10.

2. Yusuf S, Reddy S, Ounpuu S, Anand S. Global burden of cardiovascular diseases: Part I: General considerations, the epidemiologic transition, risk factors, and impact of urbanization. Circulation. 2001;104:2746-53.

3. Reddy KS, Yusuf S. Emerging epidemic of cardiovascular disease in developing countries. Circulation. 1998;97:596601.

4. Bonow RO. World Heart Day 2002: The international burden of cardiovascular disease: Responding to the emerging global epidemic. Circulation. 2002; 106:1602-05.

5. Pearson T. AHA Guidelines for primary prevention of cardiovascular disease and stroke: 2002 update: Consensus panel guide to comprehensive risk reduction for adult patients without coronary or other atherosclerotic vascular diseases. Circulation. 2002;106:388-91.

6. Cushman WC. The burden of uncontrolled hypertension: morbidity and mortality associated with disease progression. $J$ Clin Hypertens. 2003;5(suppl 2):14-22.

7. Grundy S, Benjamin I, Burke G, Chait A, Eckel R, Howard BV, et al. Diabetes and cardiovascular disease: a statement for healthcare professionals from the American Heart Association. Circulation. 1999;100:1134-46.

8. Levine G, Keaney J, Vita J. Cholesterol reduction in cardiovascular disease. Clinical benefits and possible mechanisms. N Engl J Med. 1995;332:512-21.

9. Hubert HB, McNamara PM, Castelli WP. Obesity independent risk factor for cardiovascular disease: a 26-year follow participants in the Framingham Heart Study. Circulation. 1983;67: 968-77.

10. Ross R. Atherosclerosis - an inflammatory disease. N Engl J Med. 1999; 340:115-26.

11. Thompson PD, Buchner D, Pinã IL, et al. Exercise and physical activity in the prevention and treatment of atherosclerotic cardiovascular disease. Arterioscler Thromb Vasc Biol. 2003;23(8):42-49.

12. Rabelo LMV, Viana RM, Schimith MA, Patin RV, et al. Fatores de risco para doença aterosclerótica em estudantes de uma Universidade Privada em São Paulo - Brasil. Arq Bras Cardiol. 1999;72:569-74.

13. Rodrigues ESR, Cheik NC, Mayer AF. Nível de atividade física e tabagismo em universitários. Rev Saúde Pública. 2008; 42:672-78.

14. Correia BR, Cavalcante E, Santos E. A prevalência de fatores de risco para doenças cardiovasculares em estudantes universitários. Rev Bras Clin Med. 2010;(8):25-9.

15. Martins MCC, Ricarte IF, Rocha CHL, Maia RB, Silva VB, Veras $A B$, Souza Filho MD. Pressão arterial, excesso de peso e nível de atividade física em estudantes de universidade pública. Arq Bras Cardiol. 2010;95:192-9.

16. Simão M, Hayashida M, Santos CB, Cesarino EJ, Nogueira MS. Hipertensão arterial entre universitários da cidade de Lubango, Angola. Rev Latino-Am Enfermagem. 2008; 16: 672-8.

17. Zemdegs J, Corsi L, Coelho LC, Pimentel GD, Hirai AT, Sachs A. Lipid profile and cardiovascular risk factors among first-year Brazilian university students in São Paulo. Nutr Hosp. 2011; 26:553-9.

18. Silva GSF, Bergamaschine R, Rosa M, Melo C, Miranda R, Bara Filho M. Avaliação do nível de atividade física de estudantes de graduação das áreas saúde/biológica. Rev Bras Med Esporte. 2007;13:39-42.

19. Ministério da Saúde. Universidade Federal de Goiás. Centro Colaborador em Alimentação e Nutrição da Região CentroOeste. Antropometria: manual de técnicas e procedimentos, vigilância nutricional. 2a ed. Goiânia; 2003.

20. Obesity: preventing and managing the global epidemic: report of a WHO consultation on obesity. Geneva; World Health Organization. Division of Noncommunicable Diseases. Programme of Nutrition Family and Reproductive Health.1998;

21. Furusawa EA, Franqueline M, Ruiz O, Saito MI, Koch VH, Paulo S. Avaliação do monitor de medida de pressão arterial Omron 705-CP para uso em adolescentes e adultos jovens. Arq Bras Cardiol. 2005;84:367-70.

22. Vera-Cala LM, Orostegui M, Valencia-Angel LI, Bautista LE. Precisão do aparelho Omron HEM-705 CP na medida de pressão arterial em grandes estudos epidemiológicos. Arq Bras Cardiol. 2011;96:393-8.

23. Sociedade Brasileira de Cardiologia / Sociedade Brasileira de Hipertensão / Sociedade Brasileira de Nefrologia. VI Diretrizes Brasileiras de Hipertensão. Arq Bras Cardiol. 2010; 95 (supl.1):1-51.

24. Ministério da Saúde. Resolução 196/96 do Conselho Nacional de Saúde - sobre diretrizes e normas regulamentadoras de pesquisa envolvendo seres humanos. Diário Oficial de União, Brasília (DF), 10 out. 1996.

25. World Health Organization. Global strategy on diet, physical activity and health. Geneva: World Health Organization; 2008.

26. Ministério da Saúde. Secretaria de Vigilância em Saúde. Indicadores de Mortalidade. Brasil: Ministério da Saúde; 2009. Disponível em http://tabnet.datasus.gov.br/cgi/tabcgi.exe?idb 2010/a08.def.

27. Klein S, Allison DB, Heymsfield SB, et al. Waist circumference and cardiometabolic risk: a consensus statement from Shaping America's Health: Association for Weight Management and Obesity Prevention; NAASO, the Obesity Society; the American Society for Nutrition; and the American Diabetes Association. Diabetes Care. 2007;30:1647-52.

28. Wormser D, Kaptoge S, Di Angelantonio E, Wood AM, Pennells L, Thompson A, Sarwar N, Kizer JR, Lawlor DA, Nordestgaard BG, et al: Separate and combined associations of body-mass index and abdominal adiposity with cardiovascular disease: collaborative analysis of 58 prospective studies. Lancet. 2011, 377(9771):1085-95.

29. Shiroma EJ, Lee IM. Physical activity and cardiovascular health: lessons learned from epidemiological studies across age, gender, and race/ethnicity. Circulation. 2010;122:743-52.

30. Kesaniemi YA, Danforth Jr E, Jensen MD, Kopelman PG, Lefebvre P, Reeder BA. Dose-response issues concerning physical activity and health: an evidence-based symposium. Med Sci Sport Exerc. 2001;33(Suppl):S531-8.

31. Lee IM, Djoussé L, Sesso HD, Wang L, Buring JE. Physical activity and weight gain prevention. JAMA. 2010;303:1173-9.

32. Halanych JH, Safford MM, Kertesz SG, Pletcher MJ, Kim YI, Person SD; Lewis CE, Kiefe Cl. Alcohol consumption in young adults and incident hypertension: 20-year follow-up from the coronary artery risk development in young adults study. Am J Epidemiol. 2010;171:532-9.

33. Kotsis V, Stabouli S, Papakatsika S, Rizos Z, Parati G. Mechanisms of obesity-induced hypertension. Hypertens Res. 2010; 33:386-93. 